\title{
Q/Do carotid artery calcifications seen on radiographs predict stenosis in asymptomatic adults?
}

\section{EVIDENCE-BASED ANSWER}

radiographs may be more predictive of carotid stenosis in people with atherosclerotic risk factors (SOR: C, cross-sectional study). Harms outweigh benefits in screening for carotid artery stenosis in asymptomatic adults (SOR: B, multiple cohort studies); therefore, incidental radiographic carotid artery calcifications in asymptomatic patients should not prompt further testing.

\section{Evidence summary}

Mixed results, quality issues do not support screening asymptomatic patients A meta-analysis (12 observational studies; $\mathrm{n}=1002$ ) compared the diagnostic accuracy of panoramic radiography (PR) to Doppler ultrasonography (11 studies) or angiography (1 study) in detecting calcified carotid artery atheroma (CCAA). ${ }^{1}$ The studies, conducted in 8 countries, were published after 2006. Patients were 29 to 71 years old without history of stroke, endarterectomy, angioplasty, or renal disease. In a pooled analysis, the sensitivity of PR to detect CCAA was 73\% (range, 31\%-95\%; 95\% CI, 69\%-77\%), and the specificity was $72 \%$ (range, $19 \%-99 \%$; $95 \%$ CI, $68 \%-76 \%$ ). The pooled positive predictive value was $70 \%$ (range, $37 \%-95 \%$; 95\% CI, 66\%-74\%), and negative predictive value was $75 \%$ (range, $43 \%-93 \%$; $95 \% \mathrm{CI}$, 71\%-79\%). Pooled positive likelihood ratio was 2.32 (95\% CI, 1.49-3.60) and negative likelihood ratio was 0.40 (95\% CI, 0.25-0.63). Seven studies had high risk of patient selection bias, and most had methodologic limitations.

In a retrospective cohort study $(\mathrm{n}=778)$ from the United States, researchers identified carotid artery calcifications on routine den- tal radiographs in patients $\geq 55$ years old and prospectively performed duplex ultrasound (DUS) to assess for significant carotid stenosis $(\geq 50 \%){ }^{2}$ Twenty-seven patients $(3.5 \%)$ had carotid artery calcifications on radiographs, and 20 of those patients underwent DUS of bilateral carotid arteries (40 sides). Of 26 sides with calcifications on radiograph, $13(50 \%)$ had stenosis confirmed with DUS. Of the 14 sides without calcification on radiograph, 3 (21\%) had stenosis on DUS. The positive predictive value for calcification on PR predicting significant carotid stenosis was between $40 \%$ and $80 \%$.

In a cross-sectional study from Sweden, investigators sought surgical candidates for asymptomatic carotid endarterectomy and performed PRs of 1182 patients. ${ }^{3}$ Calcifications were found in 176 people; 117 of them were eligible for asymptomatic carotid endarterectomy (ages 18-74; no cancer or other serious comorbidity; and no prior stroke or transient ischemic attack) and underwent ultrasound to assess for significant carotid stenosis ( $\geq 50 \%$ ). Of the 117 participants who underwent ultrasound, 8 (6.8\%; 95\% CI, 2.2\%-11.5\%), all men, were found to have significant carotid stenosis. Compared to a sex- and age-matched ref-
Diana Cowdrey, MD; Thomas W. Hahn, MD Department of Family Medicine and Community Health, University of Wisconsin School of Medicine and Public Health, Madison

Lia Vellardita, MA Ebling Library, University of Wisconsin School of Medicine and Public Health, Madison

DEPUTY EDITOR

Richard Guthmann, MD, MPH

Advocate Illinois Masonic Family Medicine Residency doi: 10.12788/jfp.0270 
erence group $(\mathrm{n}=119)$ with no calcifications on PR, the prevalence of carotid stenosis was significantly higher in men $(12.5 \%$; $95 \% \mathrm{CI}$, 4.2\%-20.8\%) and in patients who were current smokers (19\%; 95\% CI, 0.7\%-37.4\%), were taking cholesterol medications (13.1\%; 95\% CI 4.4\%-21.8\%), and had a cardiovascular event history (15.9\%; 95\% CI, 7\%-27.2\%).

\section{Recommendations from others}

The US Preventive Services Task Force (USPSTF) and the American Academy of Family Physicians do not mention carotid screening with radiographs but recommend against screening for carotid artery stenosis in asymptomatic adults because the risks of screening outweigh the potential benefits (USPSTF grade $\mathbf{D}$; the harms outweigh the benefits) ${ }^{4,5}$

\section{Editor's takeaway}

If you see calcification of the carotid artery on an x-ray of an asymptomatic patient, ignore it. The positive and negative predictive values for carotid stenosis are poor, and you should not pursue further testing.

JFP

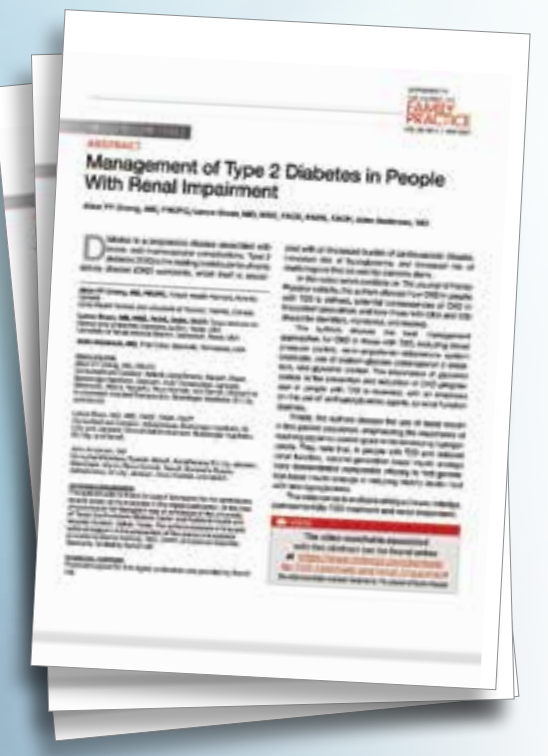

\section{Management of Type 2 Diabetes in People With Renal Impairment}

\section{Financial support for this digital publication was provided by} Sanofi US.

Diabetes is a progressive disease associated with micro- and macrovascular complications. Type 2 diabetes (T2D) is the leading contributor to chronic kidney disease (CKD) worldwide, which itself is associated with an increased burden of cardiovascular disease, increased risk of hypoglycemia, and increased risk of death beyond that caused by diabetes alone. In a series of short videos now available on The Journal of Family Practice website, the authors discuss how CKD in people with T2D is defined, potential consequences of CKD in this patient population, and how those with CKD and T2D should be identified, monitored, and treated.

Look for this supplement at https://www.mdedge.com//familymedicine 